\title{
Review of: "SARS-CoV-2 transmission during an indoor professional sporting event"
}

\author{
Mohammad Ayaz Ahmad \\ Potential competing interests: The author(s) declared that no potential competing interests exist.
}

$[1][2][3][4]$

References

1. ^Mustafa Syed Khalid, Meshari M.H. Aljohani, Naser A. Alomrani, Atif Abdulwahab A. Oyoun, et al. (2020). COVID-19 and Immune Function - "A Significant" Zinc. Orient. J. Chem, vol. 36 (6), $1026-1036$. doi:10.13005/ojc/360604.

2. ^(2021). Correction to Lancet Respir Med 2021; published online April 9. https://doi. org/10.1016/S22132600(21)00171-5. The Lancet Respiratory Medicine, vol. 9 (6), e55. doi:10.1016/s2213-2600(21)001867.

3. ^(2021). Correction to Lancet Respir Med 2021; published online April 9. https://doi. org/10.1016/S22132600(21)00160-0. The Lancet Respiratory Medicine, vol. 9 (6), e55. doi:10.1016/s2213-2600(21)001818.

4. `Charles Roberto Telles, Archisman Roy, Mohammad Rehan Ajmal, Syed Khalid Mustafa, et al. (2021). The Impact of COVID-19 Management Policies Tailored to Airborne SARS-CoV-2 Transmission: Policy Analysis. JMIR Public Health Surveill, vol. 7 (4), e20699. doi:10.2196/20699. 